\title{
Cases of Nonhealing Diabetic Neuropathic Ulcers
}

\section{Rajiv Shah}

How to cite this article: Shah R. Cases of Nonhealing Diabetic Neuropathic Ulcers. J Foot Ankle Surg (Asia-Pacific) 2016;3(2):111-116.

\section{Source of support: Nil \\ Conflict of interest: None}

\section{CASE REPORTS}

\section{Case 1: Nonhealing Diabetic Foot Ulcer}

A female aged 58 years suffering from diabetes for more than 11 years had nonhealing plantar ulcers for the past 18 months. She had diabetic neuropathy with plantar ulcerations (Fig. 1).

Pressure on an insensate foot is the cause of longstanding plantar ulcers in diabetics. Many diabetic plantar ulcers need just redistribution of weight-bearing forces. This can be done very effectively with a total contact cast. Such cast redistributes pressure and offloads the ulcerated area, giving opportunity for healing of ulcer. The principle is "one can put anything on neuropathic ulcer except pressure!"

In many diabetic patients, there is a contracture of gastrocnemius that increases plantar pressure. With a small incision, release of gastrocnemius aponeurosis at musculotendinous junction helps in stretching the muscle and thereby relieves pressure on foot. The patient also had tightness of gastrocnemius and hence she underwent treatment in the form of gastroc recession (Fig. 2). This was followed by total contact cast, which was changed every week.

As the weeks went on, the ulcer got healed (Figs 3 to 7).

Thereafter, the patient was given diabetic shoe and was also taught diabetic foot care. Ulcers healed and never recurred even at the end of follow-up after 18 months.

\section{Surgeon}

Executive Council Member, Global Foot \& Ankle Council; Vice Chairman, Asia-Pacific Foot \& Ankle Council; Past President, Indian Foot \& Ankle Society; Managing Director, Sunshine Global Hospitals Vadodara, Bharuch and Surat, Gujarat, India

Corresponding Author: Rajiv Shah, Executive Council Member Global Foot \& Ankle Council Vice Chairman, Asia-Pacific Foot \& Ankle Council; Past President, Indian Foot \& Ankle Society Managing Director, Sunshine Global Hospitals Vadodara, Bharuch and Surat Gujarat, India, e-mail: rajivortho@gmail.com

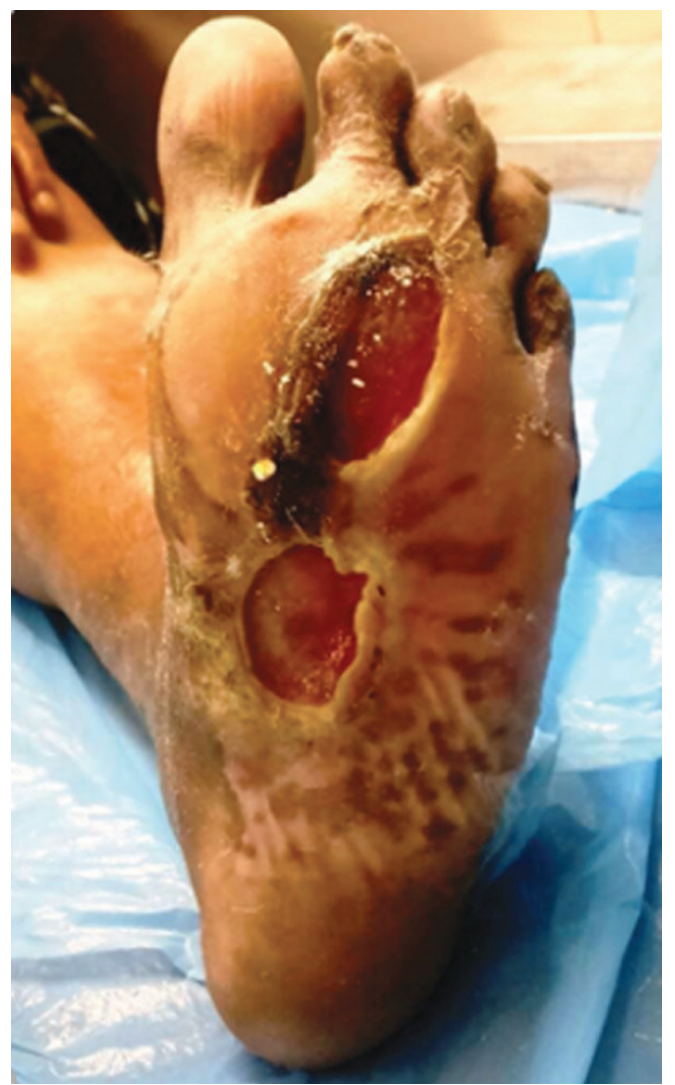

Fig. 1: Plantar aspect of left foot showing ulcers over forefoot and midfoot

Total contact cast needs to be applied with molding of arches and it should completely cover toes and needs to be changed at the end of a week to 10 days. Week by week, evaluation shows reduction in the size of ulcer. This

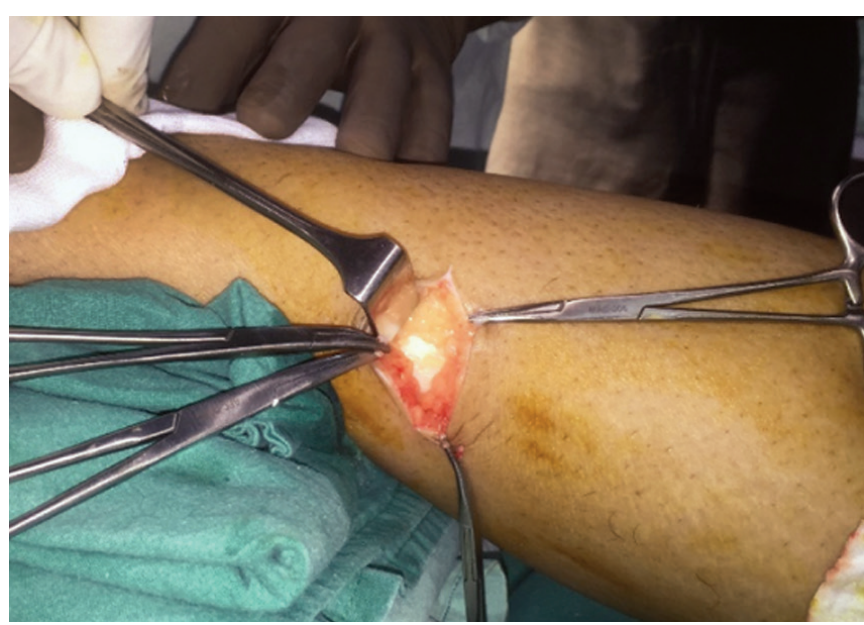

Fig. 2: Intraoperative picture showing gastrocnemius release with transverse incision 


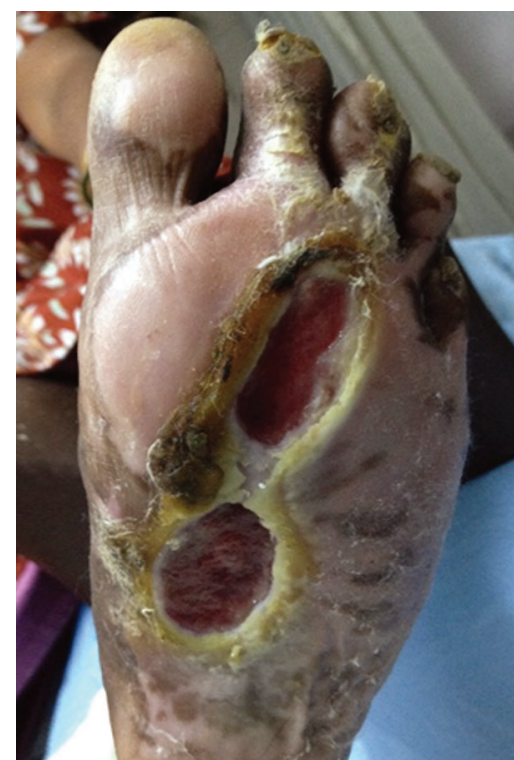

Fig. 3: Clinical picture at the end of removal of 1 st cast

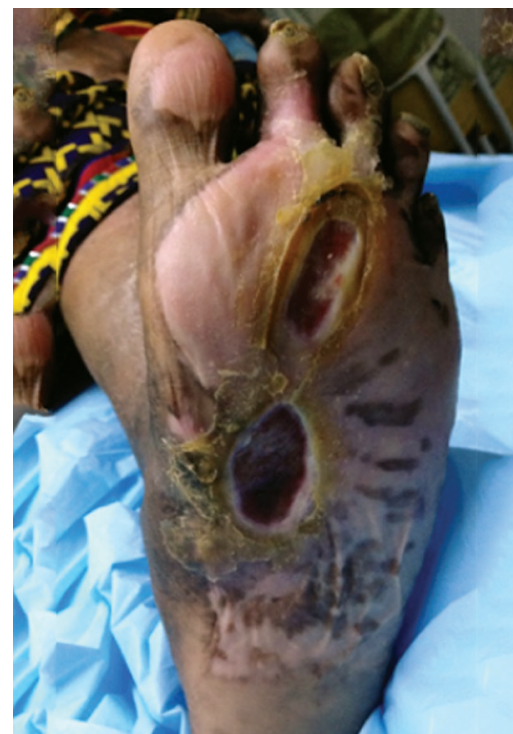

Fig. 5: Clinical picture after removal of 3rd cast

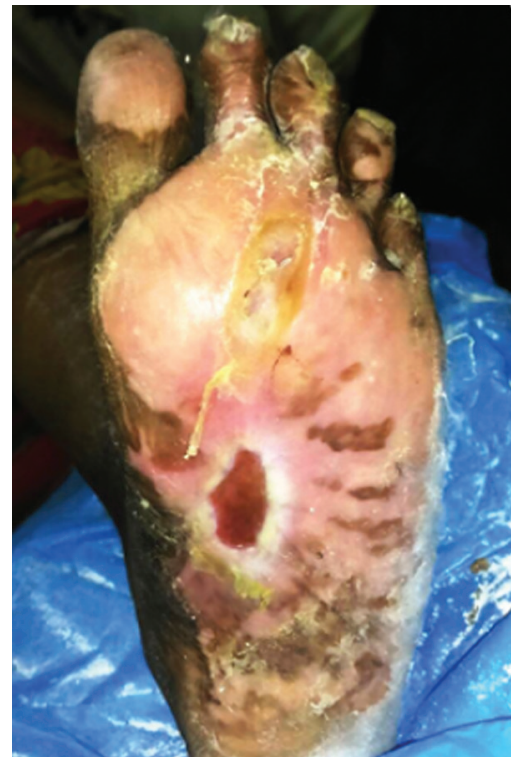

Fig. 7: Clinical picture at the end of 6 weeks after last cast

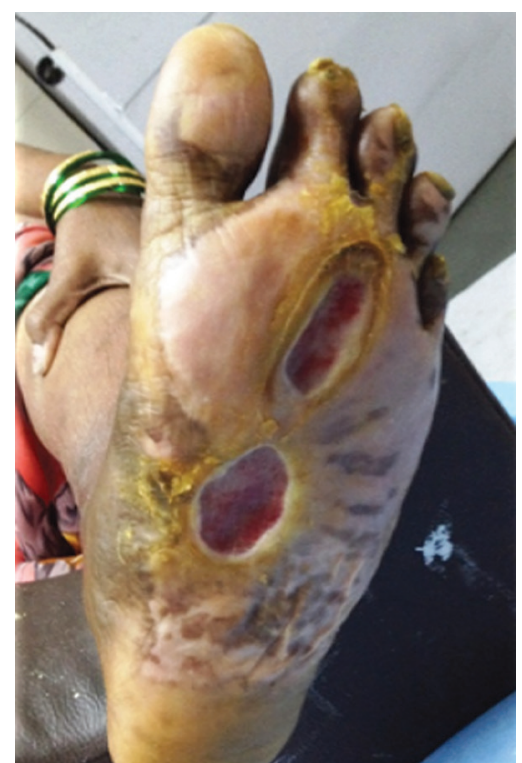

Fig. 4: Clinical picture after removal of 2nd cast

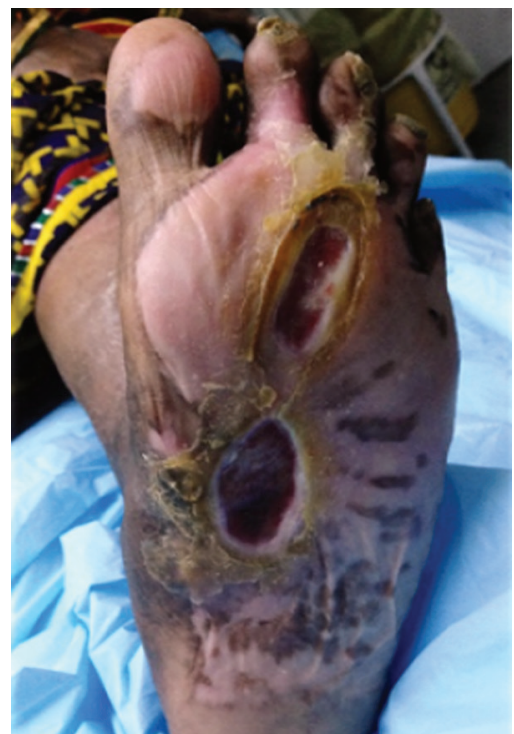

Fig. 6: Clinical picture after removal of 4 th cast

is a very important noninvasive tool for the management of plantar neuropathic ulcers.

\section{Case 2: Long-standing Nonhealing Plantar Ulcer at Base of 1st Metatarsophalangeal Joint}

A diabetic male patient aged 51 years had nonhealing plantar ulcer at the base of the 1st metatarsophalangeal (MTP) joint. On clinical examination, the patient had a drop of 1st metatarsal giving rise to plantar pressure. Plantar pressure together with neuropathy did not allow the ulcer to heal with conservative methods. Figure 8 shows the clinical picture of deformity.

Ulcers at the base of the 1st MTP joint are very common, and if not treated scientifically, they ultimately lead to amputation of great toe. Great toe being the 


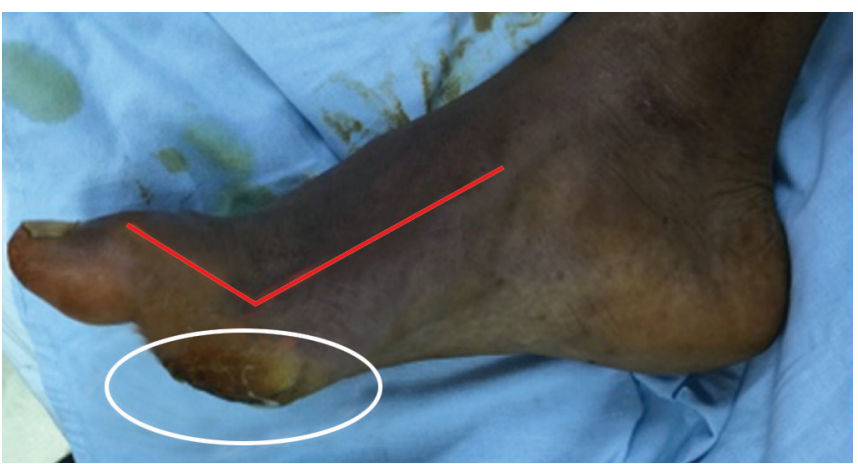

Fig. 8: Clinical picture of deformity of great toe showing drop of 1 st metatarsal with dorsiflexed hallux

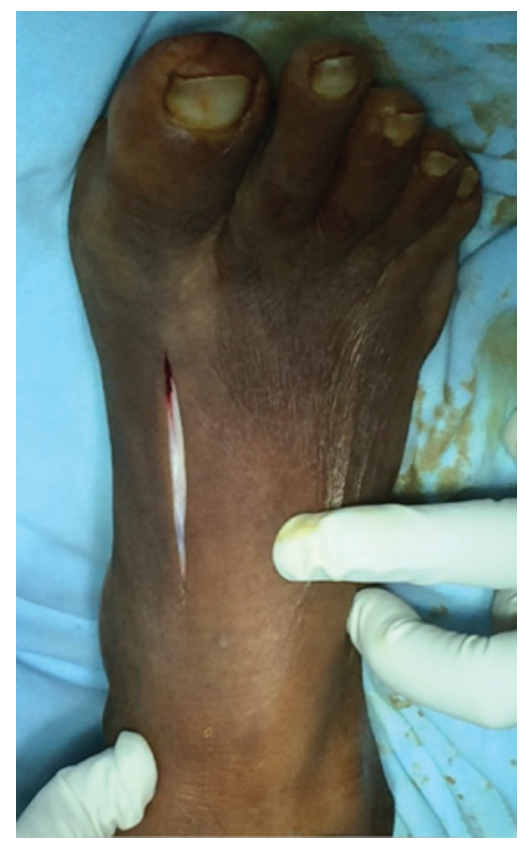

Fig. 10: Straight dorsal incision over the base of 1st metatarsal

most vital for locomotion, every effort to save it should be done. Standing radiology helps a lot in evaluation of these ulcers. Most often than not, the 1st metatarsal is fully plantarflexed leading to great pressure over plantar aspect, which in turn leads to skin breakdown and ulcer. Many a times, tightness of long great toe extensor adds to the problem. Weight-bearing X-ray of this patient confirmed the plantarflexion of 1st MTP joint generating pressure on weight bearing in a neuropathic foot (Fig. 9).

Corrective dorsiflexion wedge osteotomy of 1st metatarsal at base was done and ulcer was scrapped.

Figures 10 to 18 show the serial operative steps for the procedure.

Removal of dorsal wedge from base of 1st metatarsal helped to dorsiflex the metatarsal, in turn offloading the plantar pressure. The size of the wedge removed depends on severity of deformity but on an average wedge size of 5 to $8 \mathrm{~mm}$ works well. The osteotomy can be fixed with

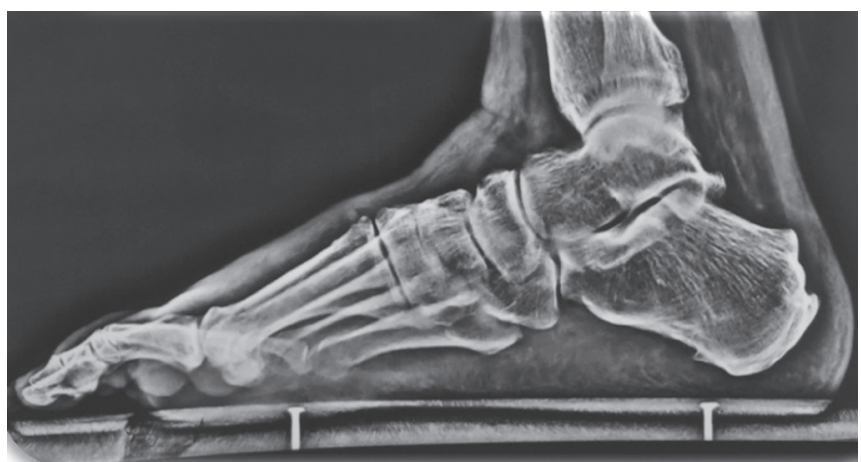

Fig. 9: Standing X-ray of the same patient showing plantarflexed 1st ray

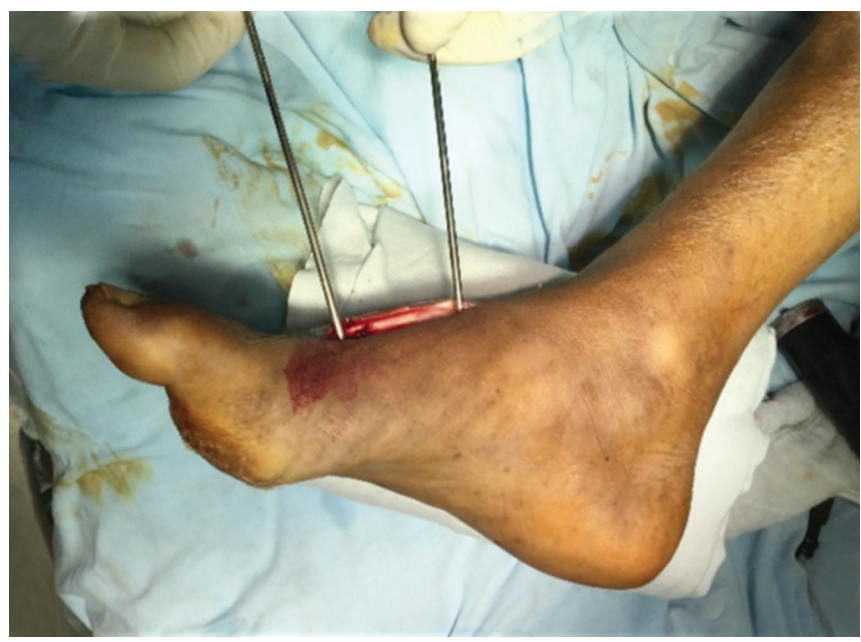

Fig. 11: Two k-wires inserted perpendicular to the axis of metatarsal and medial cuneiform to guide for osteotomy. Use of k-wires as guide to osteotomy

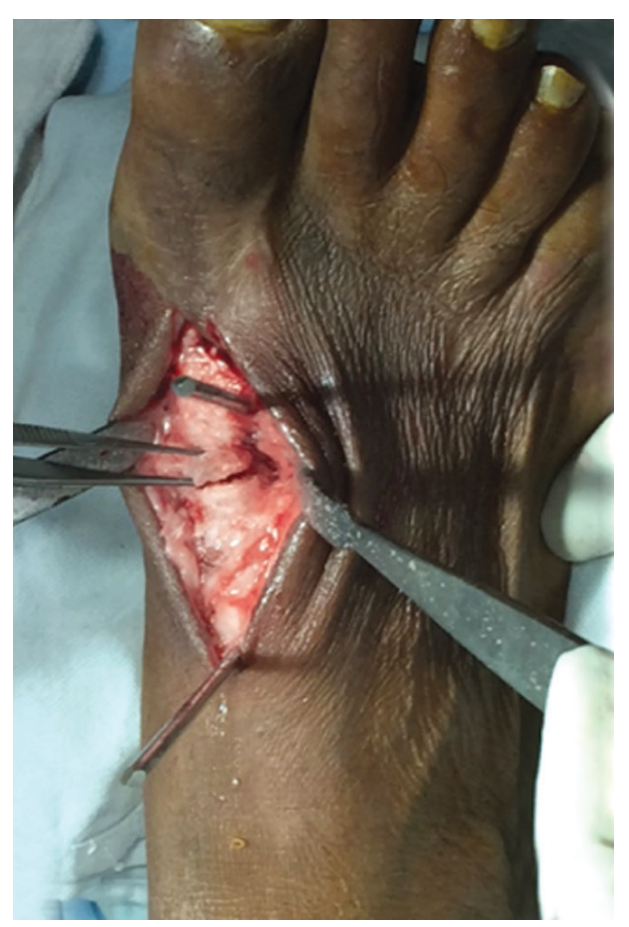

Fig. 12: Removal of dorsal wedge from the base of 1st metatarsal 


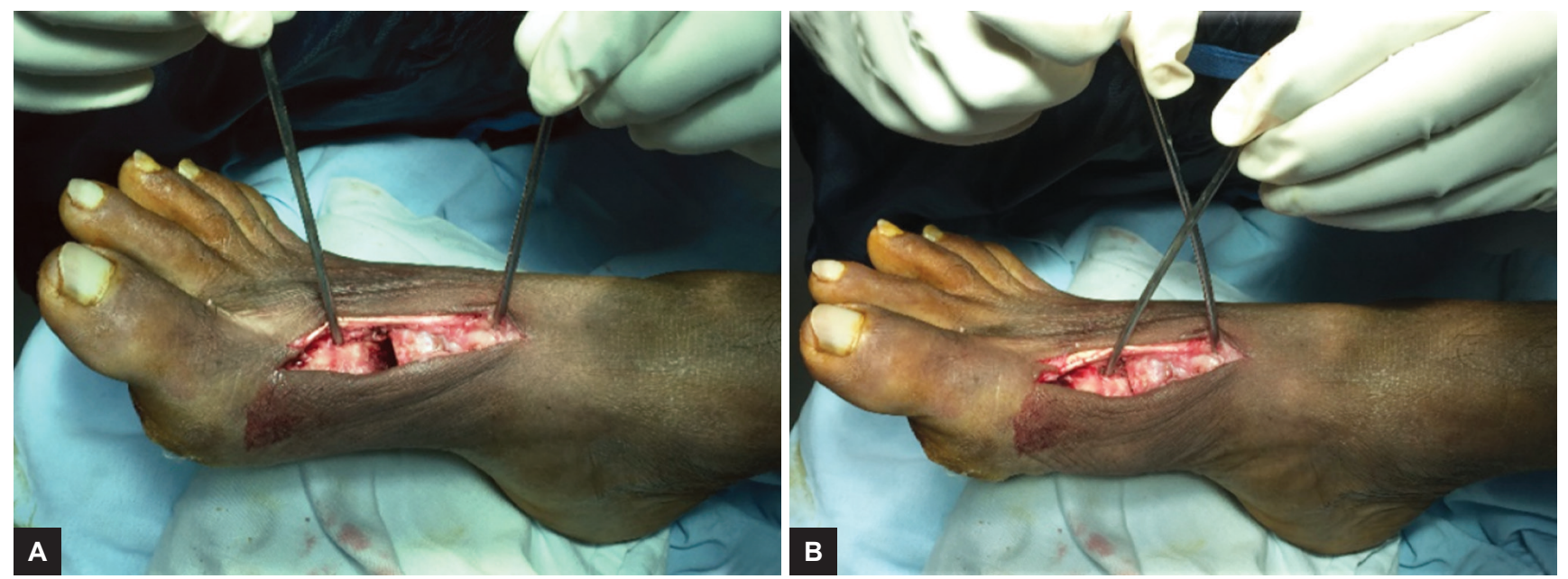

Figs $13 A$ and $B$ : Intraoperative pictures of the same case showing k-wire-aided wedge closure and deformity correction

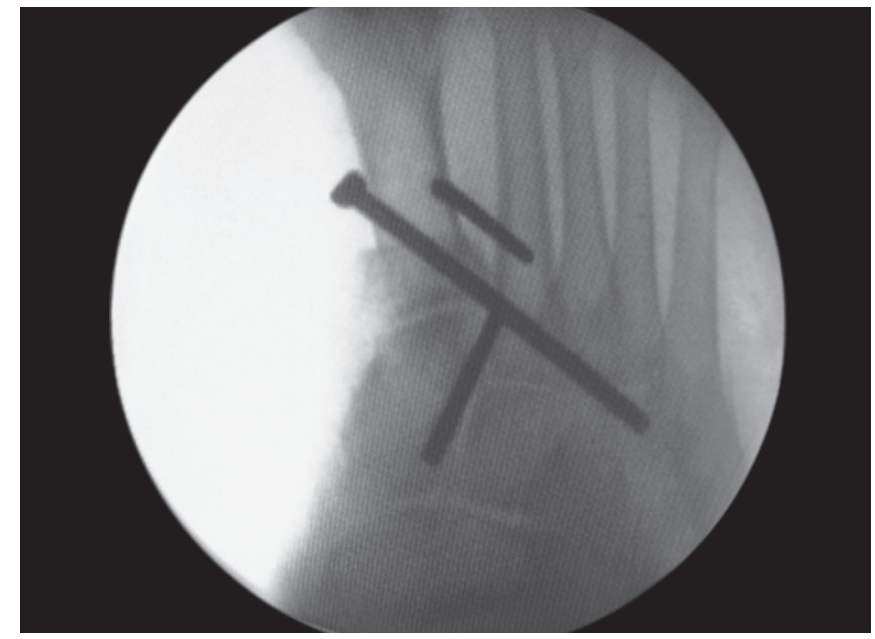

Fig. 14: Intraoperative c-arm image showing fixation of osteotomy with two screws

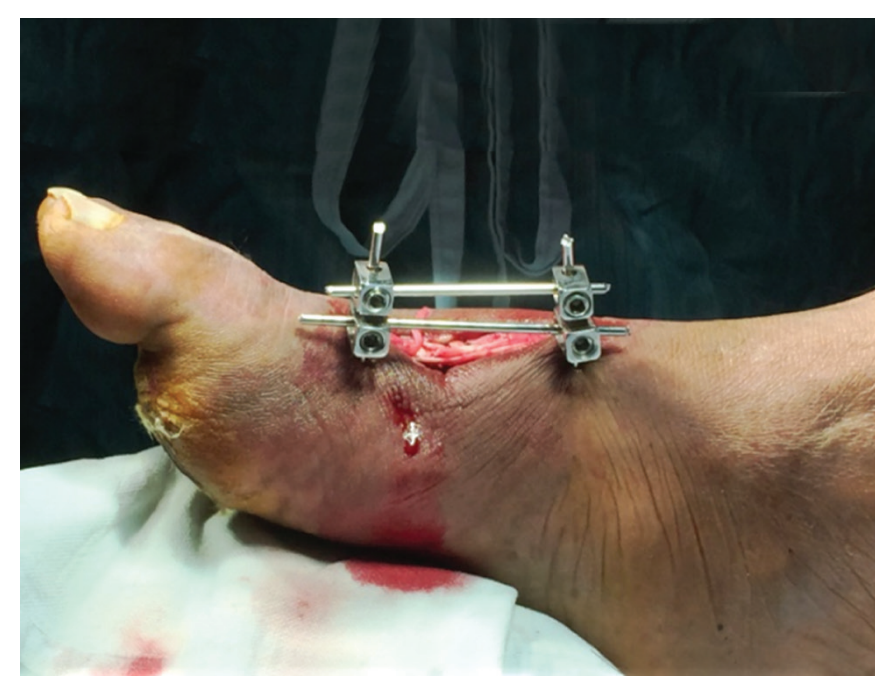

Fig. 16: Neutralizing external fixator was kept for 4 weeks to provide additional stability

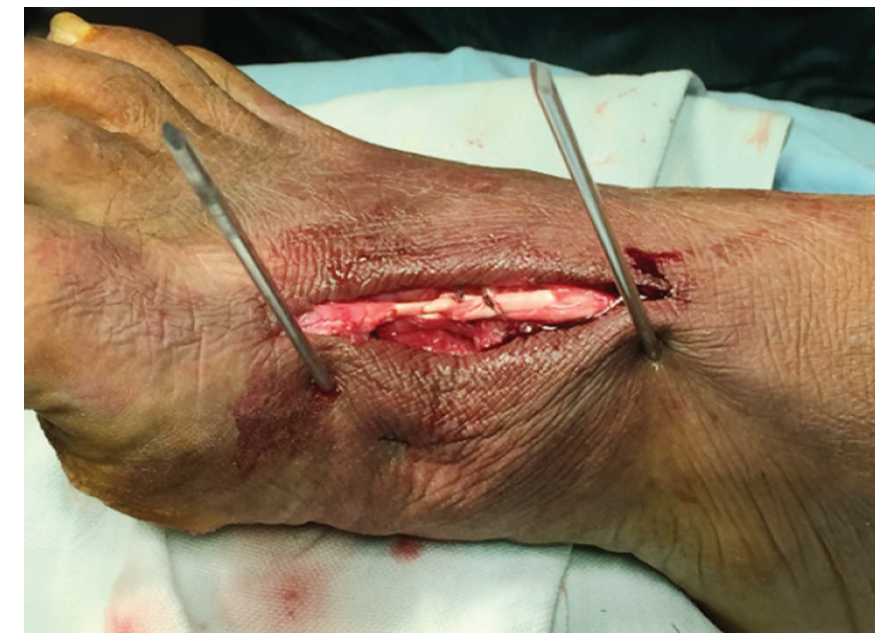

Fig. 15: Tight extensor hallucis longus was lengthened

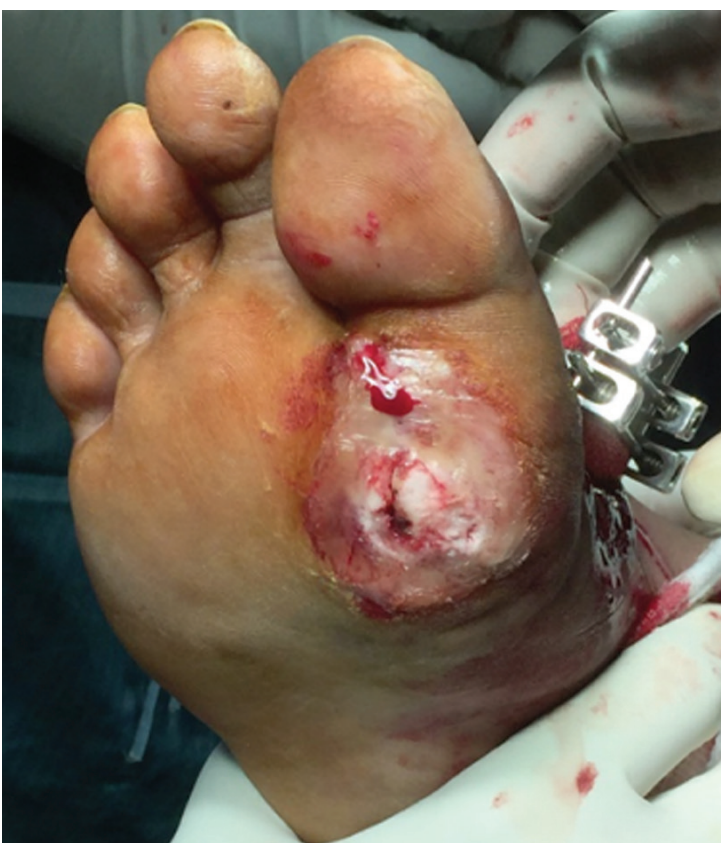

Fig. 17: Ulcer was scrapped and the final clinical picture 

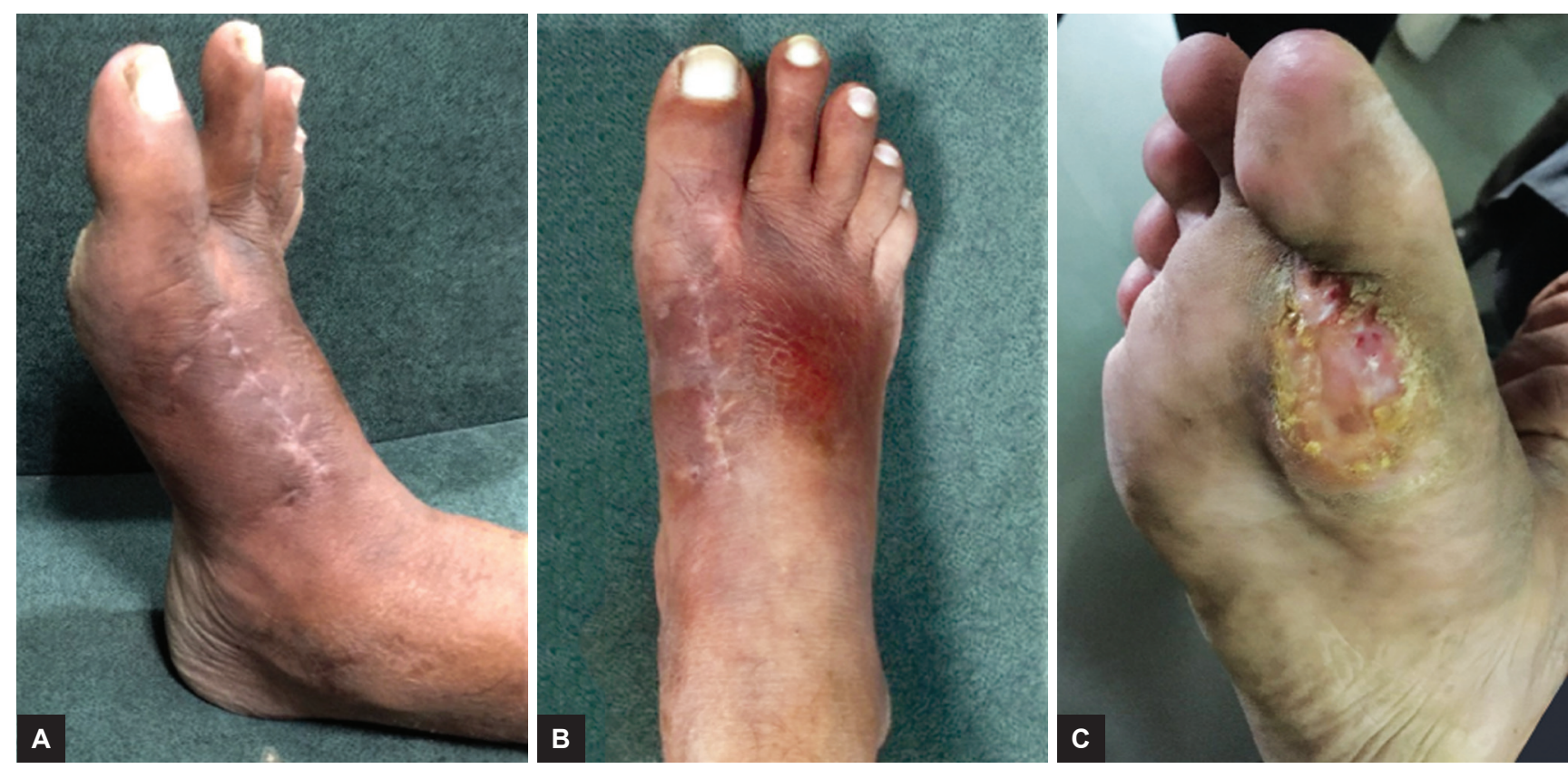

Figs 18A to C: Final clinical pictures showing correction of deformed 1st metatarsal and healing of ulcer

plate, screws, or even external fixation. The adjuvant lengthening of long extensor also helps in countering extensor force on phalanx.

\section{Case 3: Midfoot Diabetic Charcot with Nonhealing Ulcer}

An old-aged diabetic male had a nonhealing plantar ulcer at midfoot with bony prominence (Figs 19A and B).

Diabetic Charcot cases present with arch collapse at midfoot leading to rocker bottom deformity of foot. Whenever patients with such a deformity walk, skin breakage results, leading to formation of nonhealing plantar ulcer. The aim of Charcot management is to offload pressure and to maintain plantigrade foot in the orthotics or in shoes. Plantar pressure results in such cases solely due to plantar exostosis. The weight-bearing radiology gives precise idea about such exostosis. Surgeons not only need to excise this exostosis, but also need to convert it into a concave shape.

Lateral approach was taken and exostosis was excised (Figs 20 to 24).

Postsurgery, the patient underwent offloading orthotic-aided ambulation, and the ulcer healed (Fig. 25).

At surgery, a paramedian approach is preferred instead of going through the ulcer. Before completion of surgery, weight-bearing posture of foot is recreated on table to judge the adequacy of bone removal. An image check may also help. Such a surgical offloading removes plantar pressure and thereby the ulcer is healed.
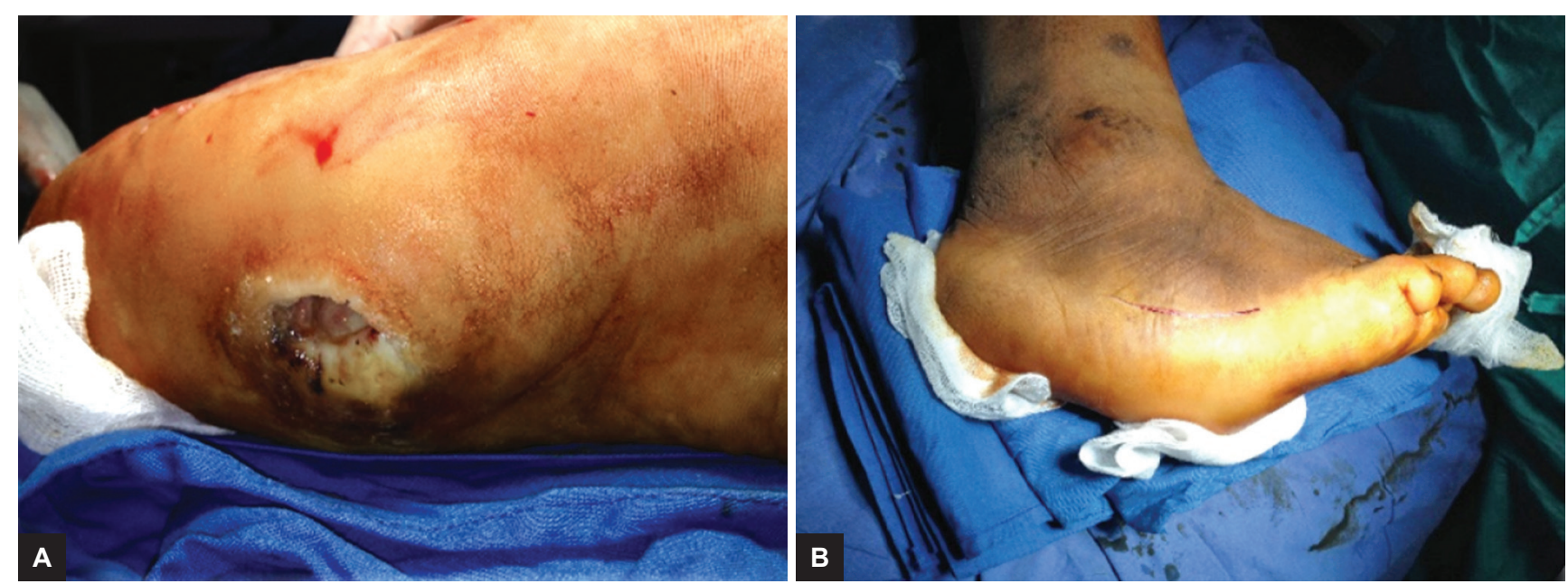

Figs 19A and B: (A) Clinical picture of plantar ulcer at midfoot; and (B) Clinical picture showing rocker bottom-shaped foot generating plantar pressure at midfoot 


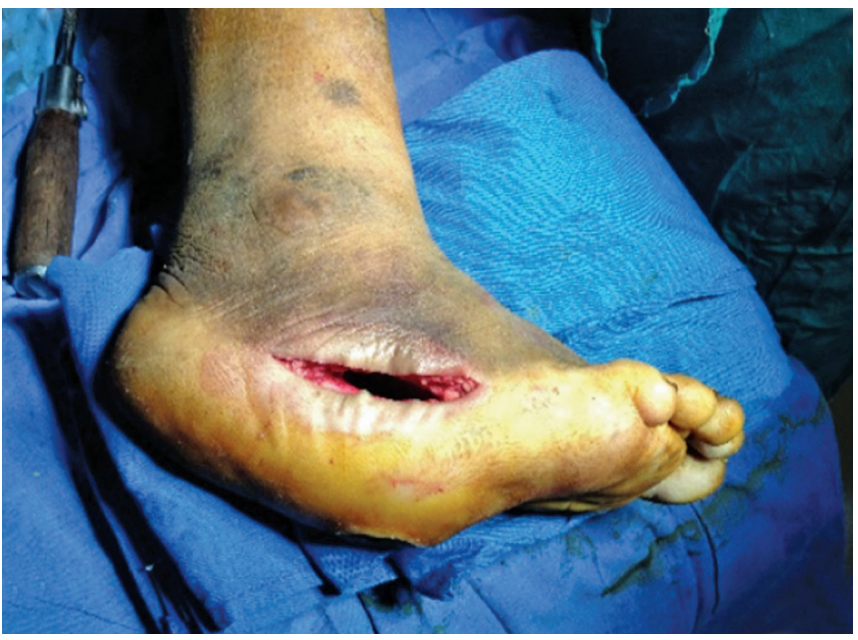

Fig. 20: Incision at the junction of dorsal and plantar skin at the lateral part of midfoot

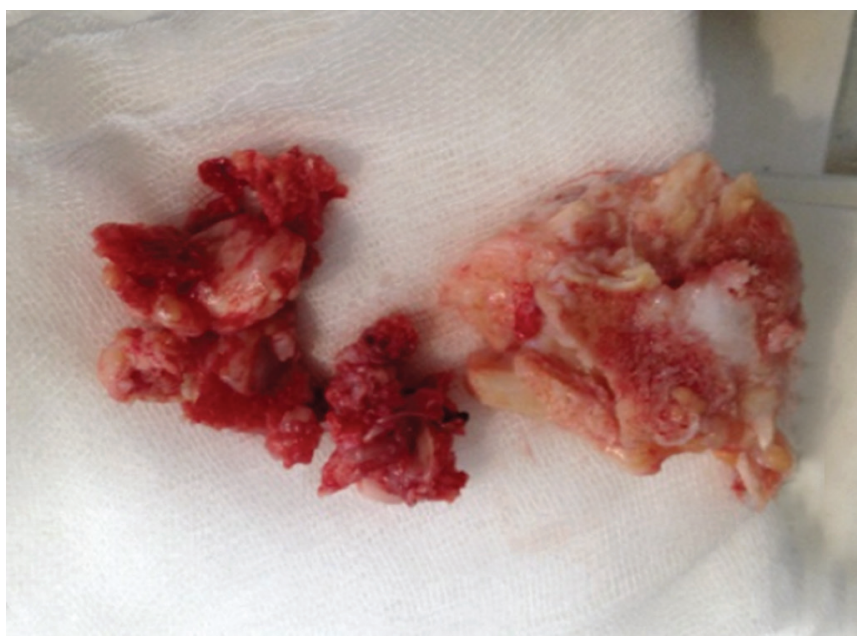

Fig. 22: Amount of bone removed is seen in this picture

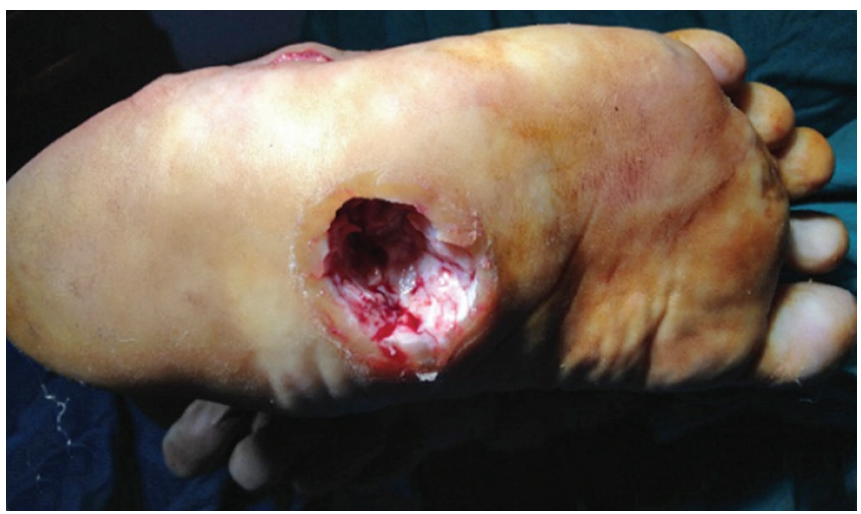

Fig. 24: Clinical picture showing scrapping of ulcer

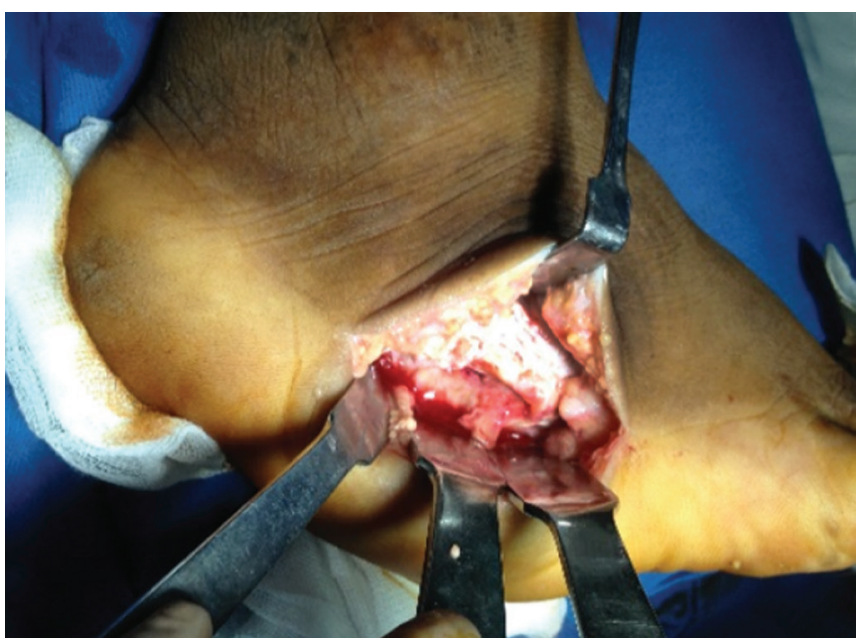

Fig. 21: Dissection showing the isolation of exostosis

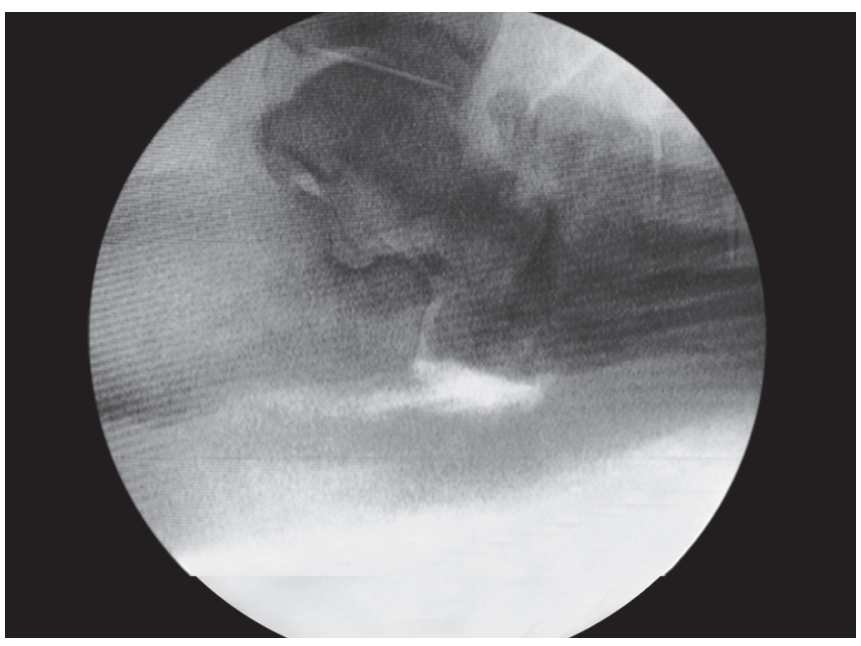

Fig. 23: Intraoperative imaging showing the amount of bone excised

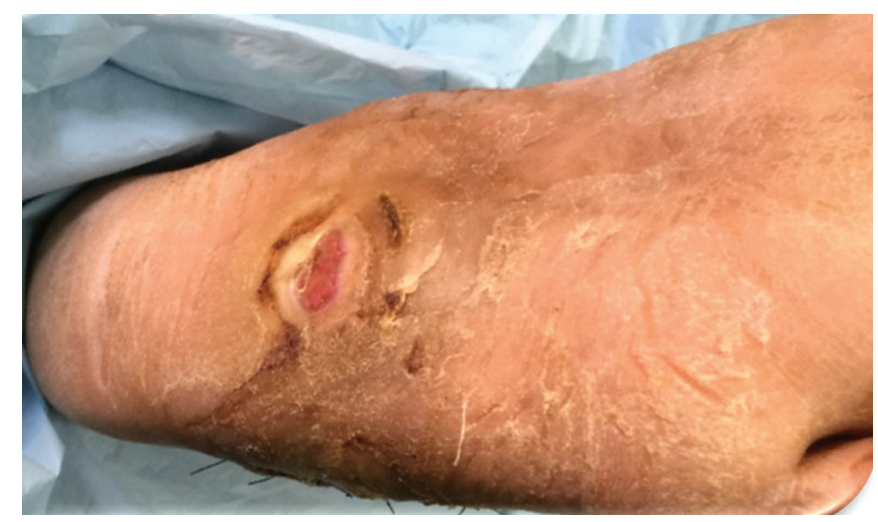

Fig. 25: Final healing of ulcer 